\title{
$\widehat{A}$ Madridge \\ madridge Journal of Dermatology \& Research \\ Interconnecting Scientific World
}

Open Access

\section{SJS/TEN Overlap Syndrome Associated with Eribulin Chemotherapy}

\author{
Timothy H Almazan and Jae Yeon Jung*
}

Division of Dermatology, City of Hope Comprehensive Cancer Center, USA

\section{Article Info}

\author{
*Corresponding author: \\ Jae Yeon Jung \\ Division of Dermatology \\ City of Hope Comprehensive Cancer \\ Center \\ 1500 East Duarte Road \\ Duarte, CA 91010 \\ USA \\ Tel: 626-256-4673, ext: 65621 \\ E-mail: jjung@coh.org
}

\section{Received: August 5, 2016}

Accepted: October 4, 2016

Published: October 7, 2016

Citation: Almazan TH, Jung JY. SJS/TEN Overlap Syndrome Associated with Eribulin Chemotherapy. Madridge J Dermatol Res. 2016; 1(1): 8-10.

doi: $10.18689 / \mathrm{mjdr}-1000102$

Copyright: @ 02016 The Author(s). This work is licensed under a Creative Commons Attribution 4.0 International License, which permits unrestricted use, distribution, and reproduction in any medium, provided the original work is properly cited.

Published by Madridge Publishers

\section{Introduction}

Eribulinmesylate is an antineoplastic, non-taxane microtubule inhibitor with emerging use as monotherapy in patients with advanced breast cancer refractory to anthracycline or taxanebased chemotherapies. Here, we present an overlap of Steven's Johnson syndrome and toxic epidermal necrolysis (SJS/TEN) that occurred secondary to eribulin administration.

\section{Case}

A woman in her 50s with a history of ER positive, PR positive, HER2 negative breast cancer complicated by liver and bony metastases presented to the City of Hope Medical Center for continued care. The patient's disease was refractory to hormonal therapy (letrozole, exemestane), as well as capecitabine and taxol. She was then switched to eribulin monotherapy. The patient received three cycles of intravenous eribulin, spaced one month apart. Dosing of the first two cycles was $1.12 \mathrm{mg} / \mathrm{m}^{2}$ and the third cycle was dose-reduced at $0.84 \mathrm{mg} / \mathrm{m}^{2}$.

Approximately one and a half months after initial eribulin administration, the patient noticed the appearance of a morbilliform rash involving her trunk and thighs. Review of systems was significant for intermittent fevers ranging from $37.8-38.0^{\circ} \mathrm{C}$. She denied any other infectious symptoms including cough, night sweats, diarrhea, or dysuria.

Upon hospital presentation, she was noted to be febrile at $38.3^{\circ} \mathrm{C}$ with a heart rate of 117 beats/min. Physical exam was significant for an erythematous, morbilliform rash with confluent papules and plaques on the trunk, back, and thighs, with sparing of the palms and soles. The rash involved approximately $60 \%$ of the body surface area. Mucosal involvement was observed in the mouth, but not the conjunctiva or anal region. Skin was exquisitely tender and pruritic. The Nikolsky's sign was negative. Labs were notable for elevated AST and ALT (similar to the patient's baseline, and attributed to known hepatic metastases), pancytopenia, and hypoalbuminemia. A punch biopsy of the left arm was obtained and revealed morphologic features of an erythema multiforme-like hypersensitivity reaction.

Concomitant medications were extensively reviewed. The patient had been taking a stable regimen of famotidine, metoclopramide, mirtazapine, furosemide, spironolactone, lorazepam, and oxycodone. There was no administration of antibiotics prior to the rash.

Based on a history of recent chemotherapy administration, morphology of the rash, results of her punch biopsy, and a negative infectious work-up, the patient's clinical presentation was thought to represent a drug-induced rash from eribulin. No further eribulin was administered. The patient receive dempiric antibiotic coverage with cefepime, vancomycin, and meropenemupon admission to the hospital, but did not demonstrate any improvement. Antibiotics were stopped, and she was initiated onhigh dose oral prednisone and completed 4 days of intravenous immunoglobulin (IVIG) 
therapy. She was continued on a prednisone taper as an outpatient. On a follow-up clinic visit one month postdischarge, the patient's rash had resolved completely. Occasional hyperpigmented patches had remained on the trunk.

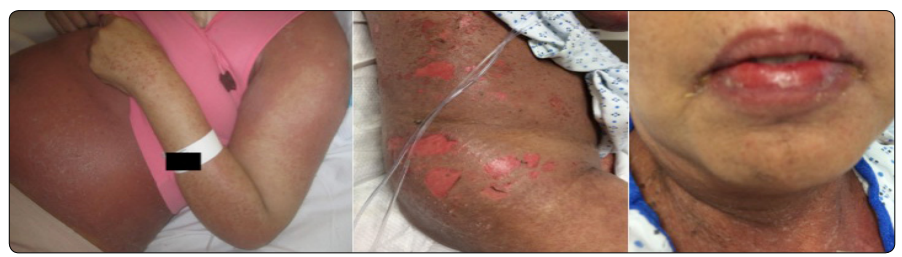

Figure 1: A woman in her 50s presenting with an erythematous, morbilliform rash with confluent papules and plaques on the trunk, back, and thighs, affecting approximately $60 \%$ BSA. Skin sloughing and mucocutaneous involvement present.

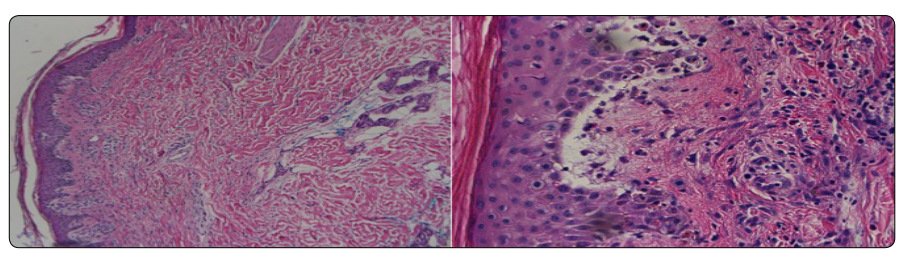

Figure 2: Histology showing keratinocyte apoptosis and lymphohistiocytic inflammatory infiltrate in the superficial dermis.

\section{Discussion}

Eribulin is a microtubule-destabilization agent that induces mitotic arrest and apoptosis in cancer cells. Similar to other tubulin-targeting drugs (such as paclitaxel and vinblastine), neuropathy is a frequent adverse symptom associated with eribulin [1]. Cutaneous manifestations from eribulin have not been commonly reported, and no prior case reports have described its possible association with SJS/TEN. In a phase I trial of eribulin, the most common eribulin-related adverse events included fatigue (present in $53 \%$ of patients), nausea (41\%), and anorexia (38\%). Eribulin-related grade 3 and 4 toxicities were noted and were comprised of neutropenia (19\%), fatigue (13\%), anorexia (3\%), anemia (3\%) and vomiting (3\%) [11].

Eribulin was shown in a phase III study to improve overall survival in heavily pre-treated patients with metastatic breast cancer. In this study, grade 3 or 4 neutropenia was experienced by 45 percent of patients receiving the drug. Palmar-plantar erythrodysaesthesia was noted in $1 \%$ of patients, and no reports of Steven's Johnson syndrome or toxic epidermal necrolysis were reported [2]. In a small group of patients $(n=6)$ receiving concomitant eribulin and cyclophosphamide for refractory breast cancer, $33 \%$ reported an unspecified rash [3].

SJS/TEN is characterized by a severe, potentially lifethreatening mucocutaneous reaction resulting in necrosis and detachment of the epidermis. Drug hypersensitivity reactions are putative causes in over $80 \%$ of cases. The most common drugs or drug classes associated with SJS/TEN include aromatic anticonvulsants, antibacterial sulfonamides, allopurinol, lamotrigine, nevirapine, and oxicam NSAIDs $[4,5]$. Other causative agents include infection with Mycoplasma pneumonia and dengue virus, cytomegalo virus reactivation, and administration of contrast medium [6]. Chemotherapy regimens have not been classically associated with SJS/TEN, but have been described in rare case reports. For example, 3 cases of SJS/TEN have been reported in association with cetuximab in a head and neck cancer patient [7].

The mortality rate from SJS/TEN is nearly 4 times as high in patients with malignancies compared to patients without [8]. Additionally, patients who received recent or ongoing chemotherapy hadan increased mortality from SJS/TEN. Other risk factors for developing SJS/TEN include a history of HIV or systemic lupus erythematosus [9].

The typical timing of SJS/TEN presentation after a causative drug administration is wide and may range between 7 days and 8 weeks (mean time of onset ranging from 6 days to 2 weeks). This is consistent with our patient's presentation of her rash, which occurred one and a half months after initial drug administration. In contrast, TEN may develop within a few hours if an implicated drug is re-administered [10]. SJS/ TEN typically results in symmetric confluent macules, extensive blisters, erosions, and denudation of the epidermis in sheets. In TEN, necrosis of the conjunctiva, trachea, bronchi, gut, and kidneys may be observed [10].

On the differential diagnosis, we considered bullous erythema mutiforme, though this was less likely given the patient's prominent mucosal involvement. Drug reaction with eosinophils and systemic symptoms (DRESS) was considered, but also less likely given a lack of systemic symptoms or organ insufficiency.

\section{Conclusion}

SJS/TEN is a life-threatening mucocutaneous reaction that typically is not associated with chemotherapy regimens. We present a case of SJS/TEN where the drug eribulin was felt to be causative. Despite high mortality of TEN (30\%), our patient was able recover successfully, likely due to prompt recognition of the SJS/TEN syndrome, discontinuation of the causative drug, and prompt treatment with high dose steroids and IVIG. SJS/TEN should be considered in any patient presenting with an extensive, desquamative rash that recently received eribulin chemotherapy.

\section{References}

1. Cortes J, Montero AJ, Gluck S. Eribulin mesylate, a novel microtubule inhibitor in the treatment of breast cancer. Cancer Treat Rev. 2012; 38(2):143-51. doi: 10.1016/j.ctrv.2011.03.006

2. Cortes J, O'Shaughnessy J, Loesch D, et al. Eribulin monotherapy versus treatment of physician's choice in patients with metastatic breast cancer (EMBRACE): a phase 3 open-label randomised study. Lancet. 2011 377(9769):914-23. doi: http://dx.doi.org/10.1016/S0140-6736(11)60070-6

3. Truong TG, Pelayo M, Grabowsky JA, et al. Phase lb study of eribulin (ERB) and cyclophosphamid (CTX) in metastatic breast cancer (MBC). ASCO Annual Meeting. Abstract 1095. 2013.

4. Mockenhaupt M, Viboud C, Dunant A, et al. Stevens-Johnson syndrome and toxic epidermal necrolysis: assessment of medication risks with emphasis on recently marketed drugs. The EuroSCAR-study. J Invest Dermatol. 2008;128(1):35-44. doi: 10.1038/sj.jid.5701033 
5. Halevy S, Ghislain PD, Mockenhaupt M, et al. Allopurinol is the most common cause of Stevens-Johnson syndrome and toxic epidermal necrolysis in Europe and Israel. J Am Acad Dermatol. 2008;58(1):25-32. doi: 10.1016/j.jaad.2007.08.036

6. Husain Z, Reddy BY, Schwartz RA. DRESS syndrome: Part I. Clinical perspectives. J Am Acad Dermatol. 2013;68(5):693 e1-14; quiz 7068. doi: 10.1016/j.jaad.2013.01.033.

7. Urosevic-Maiwald M, Harr T, French LE, Dummer R. Stevens-Johnson syndrome and toxic epidermal necrolysis overlap in a patient receiving cetuximab and radiotherapy for head and neck cancer. Int J Dermatol. 2012;51(7):864-7. doi: 10.1111/j.1365-4632.2011.05356.x.

8. Gravante G, Delogu D, Marianetti M, Esposito G, Montone A. Toxic epidermal necrolysis and Steven-Johnson syndrome in oncologic patients. Eur Rev Med Pharmacol Sci. 2007;11(4):269-74.
9. Mittmann N, Knowles SR, Koo $M$, Shear NH, Rachlis A, Rourke SB. Incidence of toxic epidermal necrolysis and Stevens-Johnson Syndrome in an HIV cohort: an observational, retrospective case series study. Am J Clin Dermatol. 2012;13(1):49-54. doi: 10.2165/11593240-00000000000000.

10. Schwartz RA, McDonough PH, Lee BW. Toxic epidermal necrolysis: Part I. Introduction, history, classification, clinical features, systemic manifestations, etiology, and immunopathogenesis. J Am Acad Dermatol. 2013;69(2):173 e1-13; quiz 85-6. doi: 10.1016/j.jaad.2013.05.003.

11. Goel S. Mita AC, Mita M, et al. A phase I study of eribulinmesylate (E7389), a mechanistically novel inhibitor of microtubule dynamics, in patients with advanced solid malignancies. Clin Cancer Res. 2009; 15: pp42074212 doi: 10.1158/1078-0432.CCR-08-2429 